\title{
Desmame e Interrupção da Ventilação Mecânica*
}

\author{
Mechanical Ventilation of Weaning Interruption
}

\author{
Rosane Goldwasser ${ }^{1}$, Augusto Farias ${ }^{2}$, Edna Estelita Freitas ${ }^{3}$,
} Felipe Saddy ${ }^{4}$, Verônica Amado ${ }^{5}$, Valdelis Novis Okamoto ${ }^{6}$.

\section{RESUMO}

JUSTIFICATIVA E OBJETIVOS: Em 2000, foi publicado o II Consenso Brasileiro de Ventilação Mecânica. Desde então, o conhecimento na área da ventilação mecânica avançou rapidamente, com a publicação de inúmeros estudos clínicos que acrescentaram informações importantes para o manuseio de pacientes críticos em ventilação artificial. Além disso, a expansão do conceito de Medicina Baseada em Evidências determinou a hierarquização das recomendações clínicas, segundo o rigor do método dos estudos que as embasaram. Essa abordagem explícita vem ampliando a compreensão e a aplicação das recomendações clínicas. Por esses motivos, a AMIB - Associação de Medicina Intensiva Brasileira - e a SBPT - Sociedade Brasileira de Pneumologia e Tisiologia - julgaram conveniente a atualização das recomendações descritas no Consenso anterior. Dentre os tópicos selecionados o desmame da Ventilação Mecânica foi um dos temas propostos. O objetivo foi descrever os pontos mais importantes relacionados ao desmame da ventilação mecânica.

MÉTODO: Objetivou-se chegar a um documento suficientemente sintético, que refletisse a melhor evidência disponível na literatura. A revisão bibliográfica baseouse na busca de estudos através de palavras-chave e em sua gradação conforme níveis de evidência. As palavras-chave utilizadas para a busca foram: weaning e mechanical ventilation.

RESULTADOS: São apresentadas recomendações quanto aos métodos de desmame utilizados, ao uso

1. Presidente da Sociedade de Terapia Intensiva do Estado do Rio de Janeiro (RJ)

2. UTI do Hospital Português, Salvador (BA)

3. Hospital Universitário Clementino Fraga Filho da Universidade Federal do Rio de Janeiro (RJ)

4. Unidade de Terapia Intensiva Hospital Copa Dor e Hospital PróCardíaco (RJ)

5. Professor Adjunto da Universidade de Brasília (DF)

6. UTI do Hospital AC Camargo (SP)

(C)Associação de Medicina Intensiva Brasileira, 2007 de protocolos e a identificação dos pacientes através do teste de ventilação espontânea.

CONCLUSÕES: A liberação do paciente do ventilador é um momento muito importante da ventilação mecânica. A utilização de protocolos para reconhecimento dos pacientes aptos a serem extubados contribui com a redução do tempo de internação dos pacientes em unidade de terapia intensiva (UTI).

Unitermos: Desmame do Ventilador, Ventilação Mecânica, Teste de ventilação espontânea, Traqueostomia.

\section{SUMMARY}

BACKGROUND AND OBJECTIVES: The II Brazilian Consensus Conference on Mechanical Ventilation was published in 2000. Knowledge on the field of mechanical ventilation evolved rapidly since then, with the publication of numerous clinical studies with potential impact on the ventilatory management of critically ill patients. Moreover, the evolving concept of evidence - based medicine determined the grading of clinical recommendations according to the methodological value of the studies on which they are based. This explicit approach has broadened the understanding and adoption of clinical recommendations. For these reasons, AMIB - Associação de Medicina Intensiva Brasileira and SBPT - Sociedade Brasileira de Pneumologia e Tisiologia - decided to update the recommendations of the II Brazilian Consensus. Weaning from mechanical ventilation has been one of the updated topics. This objective was described the most important topics related to weaning from mechanical ventilation.

METHODS: Systematic review of the published literature and gradation of the studies in levels of evidence, using the keywords weaning and mechanical ventilation.

RESULTS: Recommendations on the weaning from mechanical ventilation and how to select patients applying weaning protocols and spontaneous breathing test.

CONCLUSIONS: Weaning is a very important period of mechanical ventilatory support. Weaning protocols are recommended to select patients ready to be extubated. 
Key Words: Mechanical ventilation, Spontaneous breathing test, Tracheostomy, Weaning

\section{INTRODUÇÃO}

Retirar o paciente da ventilação mecânica pode ser mais difícil que mantê-lo. O processo de retirada do suporte ventilatório ocupa ao redor de $40 \%$ do tempo total de ventilação mecânica ${ }^{1,2}$. Alguns autores descrevem o desmame como a "área da penumbra da terapia intensiva" e que, mesmo em mãos especializadas, pode ser considerada uma mistura de arte e ciência ${ }^{3}$.

Apesar disso, a literatura tem demonstrado, mais recentemente, que protocolos de identificação sistemática de pacientes em condições de interrupção da ventilação mecânica podem reduzir significativamente sua duração ${ }^{4,5}$. Por outro lado, a busca por índices fisiológicos capazes de predizer, acurada e reprodutivelmente, o sucesso do desmame ventilatório ainda não chegou a resultados satisfatórios ${ }^{6}$.

Para que esses novos conceitos fossem mais bem incorporados na prática das unidades de terapia intensiva brasileiras, o capítulo que trata do desmame ventilatório foi atualizado no presente Consenso.

Quadro 1 - Graus de Recomendação

\begin{tabular}{ll}
\hline $\begin{array}{l}\text { Graus de Re- } \\
\text { comendação }\end{array}$ & Tratamento/Prevenção \\
\hline A & Revisão sistemática (com homogeneidade) de \\
& ensaios clínicos controlados e aleatórios \\
& Ensaio clínico controlado e aleatório com Inter- \\
& valo de confiança estreito \\
& Resultados terapêuticos do tipo "tudo ou nada" \\
& Revisão sistemática (com homogeneidade) de \\
B & estudos de coorte \\
& Estudo de coorte (incluindo ensaio clínico alea- \\
& tório de menor qualidade) \\
& Observação de resultados terapêuticos (outco- \\
& mes research) / estudo ecológico \\
& Revisão sistemática (com homogeneidade) de \\
& estudos caso-controle \\
& Estudo caso-controle \\
& Relato de casos (incluindo coorte ou caso-con- \\
& trole de menor qualidade) \\
Opinião de especialista sem avaliação crítica ou \\
baseada em matérias básicas (estudo fisiológico \\
D
\end{tabular}

\section{DEFINIÇÕES}

A retirada da ventilação mecânica é uma importante medida no tratamento intensivo. A utilização de diversos termos para definir este processo pode dificultar a avaliação de sua duração, dos diferentes modos e protocolos e do prognóstico. Por esse motivo, é importante a definição precisa dos termos, como se segue ${ }^{7,8}$ :

\section{Desmame}

O termo desmame refere-se ao processo de transição da ventilação artificial para a espontânea nos pacientes que permanecem em ventilação mecânica invasiva por tempo superior a $24 \mathrm{~h}$.

\section{Interrupção da Ventilação Mecânica}

O termo interrupção da ventilação mecânica refere-se aos pacientes que toleraram um teste de respiração espontânea e que podem ou não ser elegível para extubação.

O teste de respiração espontânea (método de interrupção da ventilação mecânica) é a técnica mais simples, estando entre as mais eficazes para o desmame. É realizado permitindo-se que o paciente ventile espontaneamente através do tubo traqueal, conectado a uma peça em forma de "T", com fonte enriquecida de oxigênio, ou recebendo pressão positiva contínua nas vias aéreas (CPAP) de $5 \mathrm{cmH}_{2} \mathrm{O}$, ou ventilação com pressão de suporte (PSV) de até $7 \mathrm{cmH}_{2} \mathrm{O}$.

\section{Extubação e Decanulação}

Extubação é a retirada da via aérea artificial. No caso de pacientes traqueostomizados, utiliza-se o termo decanulação.

Denomina-se re-intubação ou fracasso de extubação, a necessidade de reinstituir a via aérea artificial. A reintubação é considerada precoce quando ocorre em menos de 48h após a extubação (ou decanulação).

\section{Sucesso e Fracasso da Interrupção da Ventilação Mecânica}

Define-se sucesso da interrupção da ventilação mecânica como um teste de respiração espontânea bem sucedido. Os pacientes que obtiverem sucesso devem ser avaliados quanto à indicação de retirada da via aérea artificial.

Quando o paciente não tolera o teste de respiração espontânea, considera-se fracasso na interrupção da ventilação mecânica. No caso de fracasso o paciente deverá receber suporte ventilatório que promova repouso da musculatura. Uma revisão das possíveis causas desse fracasso deverá ser feita pela equipe assistente, bem como o planejamento da estratégia a ser adotada a seguir, nova tentativa de interrupção da ventilação mecânica ou desmame gradual. 


\section{Sucesso e Fracasso do Desmame}

Define-se sucesso do desmame a manutenção da ventilação espontânea durante pelo menos $48 \mathrm{~h}$ após a interrupção da ventilação artificial. Considera-se fracasso ou falência do desmame, se o retorno à ventilação artificial for necessário neste período.

\section{Ventilação Mecânica Prolongada}

Considera-se ventilação mecânica prolongada a dependência da assistência ventilatória, invasiva ou nãoinvasiva, por mais de $6 \mathrm{~h}$ por dia, por tempo superior a três semanas, apesar de programas de reabilitação, correção de distúrbios funcionais e utilização de novas técnicas de ventilação.

\section{IMPORTÂNCIA DE SE TRAÇAR ESTRATÉGIAS E PROTOCOLOS}

\section{Identificar Pacientes Elegíveis para o Teste de Res- piração Espontânea}

Recomendação: Devem-se estabelecer estratégias para identificar sistematicamente os pacientes elegíveis para o teste de respiração espontânea.

\section{Grau de Evidência: A}

Comentário: As diversas estratégias de desmame empregadas refletem julgamentos clínicos e estilos individualizados. Estudos aleatórios e controlados comprovaram que este empirismo aplicado ao desmame prolonga o tempo de ventilação mecânica ${ }^{4,5}$. É prioritário implementar estratégias para identificar sistematicamente os pacientes elegíveis para o teste de respiração espontânea. Isso reduz o tempo de ventilação mecânica e suas complicações. No entanto, um estudo aleatório e controlado avaliando o efeito de um protocolo de identificação sistemática de pacientes elegíveis para interrupção de ventilação mecânica não mostrou benefício dessa prática ${ }^{9}$, ao contrário de investigações prévias ${ }^{4,5}$. Tal discrepância foi atribuída às características da unidade onde o estudo foi realizado, a qual dispunha de uma equipe multiprofissional, adequada em número e treinamento, e de visitas diárias estruturadas por checklist, em que um dos itens foi justamente a elegibilidade para interrupção da ventilação mecânica ${ }^{9,10}$.

\section{Interrupção Diária da Sedação}

Recomendação: Pacientes sob ventilação mecânica recebendo sedativos, particularmente em infusão contínua, devem ter sedação guiada por protocolos e metas que incluam interrupção diária da infusão.

\section{Grau de Evidência: A}

Comentário: A administração contínua de sedativos é um preditor independente de maior duração da ventilação mecânica, maior permanência na UTI e no hospital ${ }^{11}$ Kress e col. ${ }^{12}$ conduziram um estudo aleatório e controlado com 128 pacientes para verificar o efeito da interrupção diária da sedação na duração da ventilação mecânica, tempo de estadia em UTI e tempo de internação hospitalar. Foi observada redução na mediana de duração de ventilação mecânica em 2,4 dias ( $p=$ $0,004)$ e na mediana de tempo de internação na UTI em 3,3 dias $(p=0,02)$ no grupo intervenção, comparado com o grupo em que a sedação não foi interrompida. Protocolos para desmame conduzidos por profissionais de saúde não-médicos reduzem o tempo de desmame $^{13}$ (vide capítulo Fisioterapia em UTI, neste Consenso). Dentro destes, os protocolos para manuseio de sedação e analgesia implementados por enfermeiros. Um desses protocolos mostrou uma redução na duração da ventilação mecânica em dois dias $(p=0,008)$, redução do tempo de permanência em UTI em dois dias $(p<0,0001)$ e redução na incidência de traqueostomia no grupo de tratamento $(6 \% \times 13 \%, p=0,04)^{13}$. Em determinadas situações clínicas, como utilização de miorrelaxantes, instabilidade hemodinâmica, fase aguda da síndrome do desconforto respiratório agudo e outras, a interrupção dos agentes sedativos deverá ser avaliada pela equipe.

\section{INTERRUPÇÃO DA VENTILAÇÃO MECÂNICA}

\section{Identificando Pacientes Elegíveis para o Teste de Respiração Espontânea}

Recomendação: A avaliação para iniciar o teste de respiração espontânea deve ser baseada primariamente na evidência de melhora clínica, oxigenação adequada e estabilidade hemodinâmica.

\section{Grau de Evidência: B}

Comentário: Para se considerar o início do processo de desmame é necessário que a doença que causou ou contribuiu para a descompensação respiratória encontre-se em resolução, ou já resolvida. O paciente deve apresentar-se com estabilidade hemodinâmica, expressa por boa perfusão tecidual, independência de vasopressores (doses baixas e estáveis são toleráveis) e ausência de insuficiência coronariana descompensada ou disritmias com repercussão hemodinâmica. Além disto, deverá ter adequada troca gasosa $\left(\mathrm{PaO}_{2} \geq\right.$ $60 \mathrm{mmHg}$ com $\mathrm{FIO}_{2} \leq 0,4$ e PEEP $\leq 5$ a $8 \mathrm{cmH}_{2} \mathrm{O}$ ) e ser capaz de iniciar os esforços inspiratórios ${ }^{8}$. 
Uma vez bem sucedido o teste de respiração espontânea, outros fatores deverão ser considerados antes de se proceder a extubação, tais como o nível de consciência, o grau de colaboração do paciente e sua capacidade de eliminar secreções respiratórias, entre outros, que serão discutidos a seguir.

\section{Como Fazer o Teste de Respiração Espontânea}

Demonstrou-se que um teste de respiração espontânea com duração de 30 min a duas horas foi útil para selecionar os pacientes prontos para extubação ${ }^{4,15-20}$. Esses mesmos estudos mostraram taxa de re-intubação em torno de $15 \%$ a $19 \%$ nos pacientes extubados. A desconexão da ventilação mecânica deve ser realizada oferecendo oxigênio suplementar a fim de manter taxas de saturação de oxigênio no sangue arterial $\left(\mathrm{SaO}_{2}\right)$ acima de $90 \%$. A suplementação de oxigênio deve ser feita com uma $\mathrm{FIO}_{2}$ até 0,4 , não devendo ser aumentada durante o processo de desconexão. Outros modos podem ser tentados para o teste de respiração espontânea, como a ventilação com pressão positiva intermitente bifásica (BIPAP - biphasic positive airway pressure) e a ventilação proporcional assistida (PAV proportional assist ventilation). Estes modos tiveram resultados iguais ao do tubo-T e PSV no teste de respiração espontânea ${ }^{21-23}$. Recentemente, uma comparação entre a compensação automática do tubo (ATC - automatic tube compensation) associada ao CPAP versus CPAP isolado mostrou-se favorável ao primeiro durante o teste de respiração espontânea. Houve maior identificação de pacientes extubados com sucesso com o ATC (82\% versus $65 \%)^{24}$. Protocolos computadorizados de desmame, presentes em alguns ventiladores, demonstraram abreviação do tempo de ventilação mecânica em número reduzido de ensaios clínicos aleatórios e devem ser observados em novos estudos 25,26 .

Os pacientes em desmame devem ser monitorados de forma contínua quanto às variáveis clínicas, às alterações na troca gasosa e às variáveis hemodinâmicas (Tabela 1).

A avaliação contínua e próxima é fundamental para identificar precocemente sinais de intolerância e mecanismos de falência respiratória. Caso os pacientes apresentem algum sinal de intolerância, o teste será suspenso e haverá o retorno às condições ventilatórias prévias. Aqueles pacientes que não apresentarem sinais de intolerância deverão ser avaliados quanto à extubação e observados (monitorados) por 48h na UTI. Se, após 48 horas, permanecerem com autonomia ventilatória, o processo estará concluído com sucesso. Se neste período necessitarem do retorno à ventilação mecânica serão considerados como insucesso ${ }^{27-29}$.

\section{Critérios de Interrupção (Fracasso) do Teste de Respiração Espontânea (Tabela 1)}

Tabela 1 - Parâmetros Clínicos e Funcionais para Interromper o Teste de Respiração Espontânea

\begin{tabular}{ll}
\hline Parâmetros & Sinais de intolerância ao teste \\
\hline Freqüência respiratória & $>35 \mathrm{ipm}$ \\
Saturação arterial de $\mathrm{O}_{2}$ & $<90 \%$ \\
Freqüência cardíaca & $>140 \mathrm{bpm}$ \\
Pressão arterial sistólica & $>180 \mathrm{mmHg}$ ou $<90 \mathrm{mmHg}$ \\
Sinais e sintomas & $\begin{array}{l}\text { Agitação, sudorese, alteração do } \\
\text { nível de consciência }\end{array}$ \\
\hline
\end{tabular}

ipm = inspirações por minuto; bpm = batimentos por minuto

\section{Conduta no Paciente que não Passou no Teste de Respiração Espontânea}

\section{(A) Repouso da Musculatura}

Recomendação: Os pacientes que falharam no teste inicial deverão retornar à ventilação mecânica e permanecer por $24 \mathrm{~h}$ em modo ventilatório que ofereça conforto, expresso por avaliação clínica. Neste período serão reavaliadas e tratadas as possíveis causas de intolerância.

\section{Grau de Evidência: A}

Comentário: O principal distúrbio fisiológico existente na insuficiência respiratória parece ser o desequilíbrio entre a carga imposta ao sistema respiratório e a habilidade em responder a essa demanda. Existem várias evidências para se aguardar $24 \mathrm{~h}$ antes de nova tentativa de desmame, para que haja recuperação funcional do sistema respiratório e de outras causas que possam ter levado à fadiga muscular respiratória, como o uso de sedativos, alterações eletrolíticas, entre outras. Nos pacientes que desenvolvem fadiga muscular, a recuperação não ocorre em menor período. A aplicação do teste de respiração espontânea com tubo-T duas vezes ao dia não foi benéfica em relação à sua aplicação uma vez ao dia ${ }^{16}$.

\section{(B) Nova Tentativa após 24 Horas}

Recomendação: Admitindo que o paciente permaneça elegível e que as causas de intolerância foram revistas, novo teste de respiração espontânea deverá ser realizado após $24 \mathrm{~h}$.

\section{Grau de Evidência: A}

Comentário: Há evidências de que a realização diária de teste de respiração espontânea abrevia o tempo de 
ventilação mecânica, em relação aos protocolos em que o teste não é realizado diariamente ${ }^{16}$.

\section{Conduta no Paciente que Passou no Teste de Res- piração Espontânea}

Uma vez que o paciente passou no teste de respiração espontânea, ele pode ou não ser elegível para extubação no mesmo dia, dependendo de outros fatores, listados na tabela 2.

\section{TÉCNICAS DE DESMAME}

\section{Redução Gradual da Pressão de Suporte}

O modo pressão de suporte também pode ser utilizado no desmame gradual de pacientes em ventilação mecânica. Isso pode ser feito através da redução dos valores da pressão de suporte de 2 a $4 \mathrm{cmH}_{2} \mathrm{O}$, de duas a quatro vezes ao dia, tituladas conforme parâmetros clínicos, até atingir 5 a $7 \mathrm{cmH}_{2} \mathrm{O}$, níveis compatíveis com os do teste de respiração espontânea. Esta estratégia foi estudada no desmame gradual de pacientes em ventilação mecânica em ensaios clínicos aleatórios. No estudo de Brochard e col. ${ }^{15}$, o uso da pressão suporte resultou em menor taxa de falha de desmame, quando comparado ao desmame em ventilação mandatória intermitente sincronizada e ao desmame com períodos progressivos (5 a $120 \mathrm{~min}$ ) de respiração espontânea em tubo-T. Já no estudo de Esteban e col., o desmame em pressão de suporte foi inferior ao desmame em tubo-T, em termos de duração e taxa de sucesso ${ }^{16}$.

\section{Ventilação Mandatória Intermitente Sincronizada} Recomendação: Evitar o modo ventilação mandatória intermitente sincronizada sem pressão de suporte (SIMV) como método de desmame ventilatório.

\section{Grau de Evidência: A}

Comentário: O modo ventilatório SIMV intercala ventilações espontâneas do paciente com períodos de ventilação assisto-controlada do ventilador mecânico. O desmame com este método é realizado reduzindo-se progressivamente a freqüência mandatória do ventilador artificial. Em quatro estudos prospectivos ${ }^{15,16,30,31}$, foi consenso ter sido este o método menos adequado empregado, pois resultou em maior tempo de ventilação mecânica. Na sua maioria, estes estudos utilizaram o método SIMV sem suporte pressórico. No estudo de Jounieux e col. ${ }^{30}$, o modo SIMV foi estudado com e sem PSV com tendência, porém sem significância estatística, a favorecer o desmame no grupo que usou PSV associado.

\section{Outros Modos}

Novos modos de ventilação, como volume suporte e ventilação de suporte adaptativa vêm sendo desenvolvidos, com vistas a facilitar e acelerar o desmame ventilatório. Sua eficácia, no entanto, ainda não foi comprovada em investigações amplas, quando comparado aos modos mais tradicionais de desmame ${ }^{32}$.

\section{ÍNDICES FISIOLÓGICOS PREDITIVOS DE FRACAS- SO DE DESMAME E EXTUBAÇÃO}

Recomendação: Os índices fisiológicos preditivos de desmame pouco auxiliam na decisão de iniciar ou não períodos de respiração espontânea, ou na redução da taxa de suporte ventilatório. A relação freqüência respiratória/volume-corrente (f/VT - índice de respiração rápida superficial) parece ser a mais acurada.

\section{Grau de Evidência: B}

Comentário: Os índices fisiológicos deveriam acres-

Tabela 2 - Fatores a Serem Considerados antes da Extubação

\begin{tabular}{ll}
\hline Fatores & Condição Necessária \\
\hline Evento agudo que motivou a VM & Revertido ou controlado \\
Troca gasosa & $\mathrm{PaO}_{2} \geq 60 \mathrm{mmHg}$ com $\mathrm{FIO}_{2} \leq 0,40$ e PEEP $\leq 5$ a $8 \mathrm{cmH}_{2} \mathrm{O}$ \\
Avaliação hemodinâmica & Sinais de boa perfusão tecidual, independência de vasopressores (doses baixas e \\
& estáveis são toleráveis), ausência de insuficiência coronariana ou disritmias com re- \\
& percussão hemodinâmica. \\
Capacidade de iniciar esforço inspiratório & Sim \\
Nível de consciência & Paciente desperta ao estímulo sonoro, sem agitação psicomotora \\
Tosse & Eficaz \\
Equilíbrio ácido-básico & pH $\geq 7,30$ \\
Balanço hídrico & Correção de sobrecarga hídrica \\
Eletrólitos séricos (K, Ca, Mg, P) & Valores normais \\
Intervenção cirúrgica próxima & Não \\
\hline
\end{tabular}

VM = ventilação mecânica; $\mathrm{K}$ = potássio; $\mathrm{Ca}=$ cálcio; $\mathrm{Mg}$ = magnésio; $\mathrm{P}$ = fósforo 
centar dados preditivos à avaliação clínica do desmame, resultando em redução das taxas de fracasso de desmame e extubação e menor tempo de ventilação mecânica ${ }^{33}$. Além disso, a técnica utilizada para sua obtenção deveria ser reprodutível, acurada, segura e de fácil realização. Infelizmente, nenhum índice fisiológico tem todas essas características.

Existem mais de 50 índices descritos, e apenas alguns (Tabela 3) auxiliam significativamente, com mudanças em relação à tomada de decisões clínicas quanto à probabilidade de sucesso ou fracasso de desmame ${ }^{8,34}$.

Tabela 3 - Índices Fisiológicos que Predizem o Fracasso do Desmame

\begin{tabular}{|c|c|c|}
\hline $\begin{array}{l}\text { Parâmetro } \\
\text { Fisiológico }\end{array}$ & Índices Fisiológicos & $\begin{array}{l}\text { Predizem Fracas- } \\
\text { so do Desmame }\end{array}$ \\
\hline Força & $\begin{array}{c}\text { Capacidade vital } \\
\text { Volume-corrente } \\
\text { Pressão inspiratória máxima } \\
\left(\mathrm{PI}_{\max }\right)\end{array}$ & $\begin{array}{c}<10 \text { a } 15 \mathrm{~mL} / \mathrm{Kg} \\
<5 \mathrm{~mL} / \mathrm{Kg} \\
>-30 \mathrm{cmH}_{2} \mathrm{O}\end{array}$ \\
\hline Endurância & $\begin{array}{c}\text { Ventilação voluntária máxima } \\
\mathrm{P}_{0,1} \\
\text { Padrão ventilatório } \\
\text { (freqüência respiratória) }\end{array}$ & $\begin{array}{l}>10 \mathrm{~L} / \mathrm{min} \\
>6 \mathrm{cmH}_{2} \mathrm{O} \\
\geq 35 \mathrm{com}\end{array}$ \\
\hline $\begin{array}{l}\text { Índices } \\
\text { combinados }\end{array}$ & $\begin{array}{c}\text { Freqüência respiratória / } \\
\text { volume-corrente (L) } \\
\text { f/VT }\end{array}$ & $>104 \mathrm{cpm} / \mathrm{L}$ \\
\hline
\end{tabular}

Entre os índices mensurados durante o suporte ventilatório, apenas cinco têm possível valor em predizer o resultado do desmame:

1. Força inspiratória negativa;

2. Pressão inspiratória máxima (PImax);

3. Ventilação minuto (V'E);

4. Relação da pressão de oclusão da via aérea nos primeiros $100 \mathrm{~ms}$ da inspiração $(\mathrm{P0}, 1)$ pela pressão inspiratória máxima (P0,1/PImax); e

5. CROP: complacência, freqüência, oxigenação, pressão.

Dos índices descritos, apenas os dois últimos apresentam taxas de probabilidade sugerindo aplicação clíni$\mathrm{ca}^{33}$.

Entre os índices medidos durante ventilação espontânea, a acurácia é melhor em relação aos seguintes parâmetros, quando medidos durante 1 a 3 minutos de respiração espontânea: freqüência respiratória, volume-corrente e relação freqüência / volume-corrente (f/VT - índice de respiração rápida superficial), sendo este último o mais acurado. Entretanto, mesmo estes testes estão associados a mudanças pequenas ou moderadas na probabilidade de sucesso e fracasso no desmame ${ }^{8,34}$.

\section{EXTUBAÇÃO TRAQUEAL}

\section{Uso de Corticosteróides}

Recomendação: O uso profilático de corticóides sistêmicos, para evitar estridor após extubação traqueal e a necessidade de re-intubação, não é recomendado para pacientes adultos.

\section{Grau de Evidência: B}

Comentário: Os estudos realizados até o momento não respaldam o uso de corticóide sistêmico para prevenção do estridor pós-extubação e para a necessidade de re-intubação em pacientes adultos ${ }^{35-38}$.

\section{Teste de Permeabilidade}

Recomendação: O teste de permeabilidade (escape aéreo entre via aérea e cânula traqueal após desinsuflação do balonete) pode ser usado para identificar pacientes com maior probabilidade de obstrução de via aérea, por edema ou granuloma, após a extubação traqueal, especialmente após ventilação mecânica prolongada.

\section{Grau de Evidência: C}

Comentário: A intubação traqueal pode causar inflamação e edema laríngeo, predispondo à obstrução da via aérea (manifesta clinicamente pelo estridor laríngeo), tão logo o tubo traqueal seja retirado. Essa é uma importante causa de fracasso na extubação ${ }^{39}$. $\mathrm{O}$ teste de permeabilidade consiste em medir o volume-corrente expiratório através do tubo traqueal com o balonete insuflado e a seguir desinsuflar o balonete e medir novamente o volume-corrente expiratório. Se houver escape aéreo em torno do tubo traqueal (definindo a existência de espaço entre o tubo e a via aérea), o volume-corrente expiratório será menor que o volume-corrente inspiratório, sugerindo menor probabilidade de edema laríngeo e estridor após extubação. Estudos mais antigos verificaram o escape aéreo de forma qualitativa. Mais recentemente, outros estudos quantificaram-no ${ }^{40,41}$. Miller e col. estudaram 100 intubações consecutivas e observaram estridor laríngeo em $80 \%$ dos pacientes, em que a diferença entre o volume-corrente inspiratório e o volume-corrente expiratório após (média de três medidas) foi $\leq 110 \mathrm{~mL}$. Já, $98 \%$ daqueles em que o vazamento foi $>110 \mathrm{~mL}$ não apresentaram estridor ${ }^{37}$.

O teste de permeabilidade mostrou-se útil em identificar maior risco de estridor pós-extubação traqueal em pacientes que permaneceram mais de $48 \mathrm{~h}$ intubados em UTI gerais ${ }^{40,41}$. Por outro lado, não foi um bom preditor de estridor pós-extubação traqueal em pacientes 
em pós-operatório de cirurgia cardíaca, nos quais o tempo médio de intubação traqueal foi de $12 h^{42}$.

Uma boa higiene de vias aéreas superiores, visando à prevenção de aspiração das secreções que se pode acumular na traquéia, acima do balonete, deve ser realizada.

\section{Cuidados Gerais Pré-Extubação}

Recomendação: Antes de proceder a extubação, a cabeceira do paciente deve ser elevada, mantendo-se uma angulação entre $30^{\circ}$ e $45^{\circ}$. Também é indicado que se aspire a via aérea antes de extubá-lo.

\section{Grau de Evidência: D}

Comentário: O acúmulo de secreção e a incapacidade de eliminá-la através da tosse são fatores que contribuem de maneira importante para o insucesso da extubação traqueal, mesmo após um teste de respiração espontânea bem sucedido ${ }^{43,44}$. Nos pacientes que apresentaram alguma dificuldade no procedimento de intubação traqueal ou que tenham importantes fatores de risco para complicações obstrutivas após extubação, pode-se optar por assegurar a via aérea pérvia, mantendo-se um trocador de cânula endotraqueal em posição por algumas horas, até que se tenha maior segurança quanto ao sucesso da extubação ${ }^{45}$.

Aspirar as vias aéreas antes da extubação tem como objetivos diminuir a quantidade de secreção nas vias aéreas baixas e retirar a secreção que se acumula acima do balonete da cânula traqueal, evitando a sua aspiração para os pulmões. A elevação da cabeceira visa, por sua vez, a diminuir a probabilidade do paciente aspirar o conteúdo gástrico. Dessa forma pretende-se diminuir as possibilidades de infecção respiratória ${ }^{46}$.

\section{TRAQUEOSTOMIA}

Recomendação: A traqueostomia precoce (até 48h do início da ventilação mecânica) em pacientes com previsão de permanecer por mais de 14 dias em ventilação mecânica reduzem a mortalidade, a pneumonia associada à ventilação mecânica, os tempos de internação em UTI e de ventilação mecânica.

\section{Grau de Evidência: B}

Comentário: Durante muito tempo não foi possível definir o papel da traqueostomia no desmame ventilatório e o momento certo de realizá-la. Não há uma regra geral em relação ao tempo que se deve realizar a traqueostomia e este procedimento deve ser individualizado. Embora haja alguma divergência de resultados, a traqueostomia diminui a resistência e o trabalho ventilatório, facilitando o desmame dos pacientes com alterações acentuadas da mecânica respiratória ${ }^{47,48}$.
Metanálise publicada em 1998 deixou claro que não existia, até então, uma definição quanto ao momento mais adequado para indicar a traqueostomia, uma vez que os estudos eram discordantes, alguns não eram aleatórios na escolha dos pacientes e nenhum era duplamente encoberto ${ }^{49}$. Entretanto, recentemente, estudo aleatório, envolvendo pacientes que presumivelmente permaneceriam intubados por mais que 14 dias (pacientes com doença neurológica de progressão lenta ou irreversível e/ou doença de via aérea superior), mostrou benefício em termos de mortalidade, incidência de pneumonia, tempo de internação em UTI e tempo de ventilação mecânica nos pacientes submetidos à traqueostomia precoce (nas primeiras $48 \mathrm{~h}$ de intubação traqueal) ${ }^{50}$. Cabe salientar, entretanto, que este trabalho não deixou claro quais foram os critérios sugestivos de maior tempo de intubação traqueal e necessidade de ventilação mecânica. Também é importante enfatizar que, mantendo boas práticas em relação à insuflação do balonete, o tubo traqueal pode ser mantido por tempo superior a três semanas sem lesão laríngea ou traqueal.

\section{OUTROS ASPECTOS ASSOCIADOS À INTERRUP- ÇÃO DA VENTILAÇÃO MECÂNICA E AO DESMAME VENTILATÓRIO}

Vários aspectos relacionados à qualidade da assistência ao paciente com insuficiência respiratória têm efeito direto na eficiência e efetividade do desmame. Alguns itens foram abordados nos demais capítulos do Consenso. Seguem algumas recomendações específicas dos processos de desmame e de interrupção da ventilação mecânica.

\section{Dispositivos Trocadores de Calor}

Recomendação: Deve-se estar atento à possível contribuição negativa dos trocadores de calor nos pacientes com falência de desmame.

\section{Grau de Evidência: A}

Comentário: Os umidificadores trocadores de calor vêm progressivamente substituindo os aquecedores dos ventiladores. Dois estudos aleatórios compararam o efeito destes dispositivos sobre parâmetros fisiológicos respiratórios. Os pacientes em uso de trocadores de calor apresentaram significativo aumento de volume-minuto, freqüência respiratória, $\mathrm{PaCO}_{2}$, trabalho da respiração, produto pressão $x$ tempo, pressão esofágica e transdiafragmática, PEEP intrínseco, acidose respiratória, além de maior desconforto respiratório ${ }^{51,52}$. 
Os autores concluíram que a presença de dispositivos trocadores de calor deve ser levada em conta nos pacientes de desmame difícil, especialmente aqueles com insuficiência ventilatória crônica.

\section{Hormônio do Crescimento}

Recomendação: Não existe recomendação para o uso de hormônio do crescimento como recurso para incrementar o desmame da ventilação.

\section{Grau de Evidência: B}

Comentário: Estudos não controlados têm sugerido possível efeito benéfico do hormônio do crescimento sobre pacientes com dificuldade de desmame ${ }^{53,54}$. Entretanto, um estudo prospectivo aleatório, controlado, encoberto, foi realizado com 20 pacientes que necessitaram de ventilação mecânica por pelo menos sete dias, devido a insuficiência respiratória aguda. O grupo intervenção usou hormônio do crescimento recombinante por 12 dias. Apesar da acentuada retenção de nitrogênio, observada no grupo intervenção, isto não se refletiu em menor duração do desmame ou melhora da força muscular ${ }^{55}$.

\section{Hemotransfusões}

Recomendação: Transfusões sangüíneas não devem ser usadas rotineiramente visando a facilitar o desmame ventilatório.

\section{Grau de Evidência: B}

Comentário: Não há evidência de que uma estratégia liberal referente a transfusões sangüíneas (reposição de glóbulos em pacientes com hemoglobina $\leq 9 \mathrm{mg} /$ $\mathrm{dL}$ ) tenha reduzido o tempo de duração mecânica em uma população heterogênea ${ }^{56}$.

\section{Suporte Nutricional}

Recomendação: Dietas de alto teor de gordura e baixo teor de carboidratos podem ser benéficas em pacientes selecionados, com limitada reserva ventilatória, para redução do tempo de desmame. Entretanto, em virtude do pequeno número de estudos não se recomenda o seu uso rotineiro.

\section{Grau de Evidência: B}

Comentário: As dietas com elevado teor de gordura e baixo teor de carboidratos parecem produzir efeitos favoráveis na produção de $\mathrm{CO}_{2}$, o que pode facilitar o desmame de pacientes com limitada reserva ventilatória, inclusive em reduzir o tempo de desmame. $\mathrm{O}$ motivo da utilização das dietas de alto teor de gordura e baixo teor de carboidrato consiste em que um menor quociente respiratório pode melhorar a troca gasosa e facilitar o desmame da ventilação mecânica em pacientes com reserva ventilatória limitada. Entretanto, os estudos ainda não têm respaldo para uma recomendação mais ampla ${ }^{57,58}$.

\section{REFERÊNCIAS}

01. Esteban A, Alia I, Ibanez $\mathrm{J}$ et al - Modes of mechanical ventilation and weaning. A national survey of Spanish hospitals. The Spanish Lung Failure Collaborative Group. Chest, 1994;106:1188-1193.

02. Esteban A, Anzueto A, Frutos F et al - Characteristics and outcomes in adult patients receiving mechanical ventilation: a 28-day international study. JAMA, 2002;287:345-355.

03. Milic-Emili $\mathrm{M}$ - Is weaning an art or a science? Am $\mathrm{J}$ Respir Dis, 1986;134:1107-1108.

04. Ely EW, Baker AM, Dunagan DP et al - Effect on the duration of mechanical ventilation of identifying patients capable of breathing spontaneously. N Engl J Med, 1996;335:1864-1969.

05. Kollef $\mathrm{MH}$, Shapiro $\mathrm{SD}$, Silver $\mathrm{P}$ et al - A randomized, controlled trial of protocol-directed versus physician-directed weaning from mechanical ventilation. Crit Care Med, 1997;25:567-574

06. Vallverdu I MJ - Weaning Criteria: Physiologic Indices in Different Groups of Patients. Berlin Heidelberg: Springer-Verlag; 2002.

07. Force AAST - Evidence based guidelines for weaning and discontinuing mechanical ventilatory support. Chest, 2001;120:(Suppl6):375-395.

08. Maclntyre NR, Cook DJ, Ely EW Jr et al - Evidence-based guidelines for weaning and discontinuing ventilatory support: a collective task force facilitated by the American College of Chest Physicians; the American Association for Respiratory Care; and the American College of Critical Care Medicine. Chest, 2001;120:(Suppl6):375S- 395S.

09. Krishnan JA, Moore D, Robeson C et al - A prospective, controlled trial of a protocol-based strategy to discontinue mechanical ventilation. Am J Respir Crit Care Med, 2004;169:673-678.

10. Tobin MJ - Of principles and protocols and weaning. Am J Respir Crit Care Med, 2004;169:661-662.

11. Kollef MH, Levy NT, Ahrens TS et al - The use of continuous i.v. sedation is associated with prolongation of mechanical ventilation. Chest, 1998;114:541-548.

12. Kress JP, Pohlman AS, O'Connor MF et al - Daily interruption of sedative infusions in critically ill patients undergoing mechanical ventilation. $\mathrm{N}$ Engl J Med, 2000;342:1471-1477.

13. Ely EW, Meade MO, Haponik EF et al - Mechanical ventilator weaning protocols driven by nonphysician health-care professionals: evidence-based clinical practice guidelines. Chest, 2001;120:(Suppl6):454S463S.

14. Brook AD, Ahrens TS, Schaiff R et al - Effect of a nursing-implemented sedation protocol on the duration of mechanical ventilation. Crit Care Med, 1999;27:2609-2615.

15. Brochard L, Rauss A, Benito $S$ et al - Comparison of three methods of gradual withdrawal from ventilatory support during weaning from mechanical ventilation. Am J Respir Crit Care Med, 1994;150:896-903.

16. Esteban A, Frutos F, Tobin MJ et al - A comparison of four methods of weaning patients from mechanical ventilation. Spanish Lung Failure Collaborative Group. N Engl J Med, 1995;332:345-350.

17. Esteban A, Alia I, Gordo F et al - Extubation outcome after spontaneous breathing trials with T-tube or pressure support ventilation. The Spanish Lung Failure Collaborative Group. Am J Respir Crit Care Med, 1997;156(2 Pt 1):459-465.

18. Esteban A, Alia I, Tobin MJ et al - Effect of spontaneous breathing trial duration on outcome of attempts to discontinue mechanical ventilation. Spanish Lung Failure Collaborative Group. Am J Respir Crit Care Med, 1999;159:512-518.

19. Perren A, Domenighetti G, Mauri S et al - Protocol-directed weaning from mechanical ventilation: clinical outcome in patients randomized for a 30-min or 120-min trial with pressure support ventilation. Intensive Care Med, 2002;28:1058-1063.

20. Matic I, Majeric-Kogler V - Comparison of pressure support and T-tube weaning from mechanical ventilation: randomized prospective study. Croat Med J, 2004;45:162-166. 
21. Elrazek EA - Randomized prospective crossover study of biphasic intermittent positive airway pressure ventilation (BIPAP) versus pressure support ventilation (PSV) in surgical intensive care patients. Middle East J Anesthesiol, 2004;17:1009-1021.

22. Haberthur $\mathrm{C}$, Mols $\mathrm{G}$, Elsasser $\mathrm{S}$ et al - Extubation after breathing trials with automatic tube compensation, T-tube, or pressure support ventilation. Acta Anaesthesiol Scand, 2002;46:973-979.

23. Grasso S, Puntillo F, Mascia L et al - Compensation for increase in respiratory workload during mechanical ventilation. Pressure-support versus proportional-assist ventilation. Am J Respir Crit Care Med, 2000;161:(3 Pt 1):819-826.

24. Cohen JD, Shapiro M, Grozovski E et al - Extubation outcome following a spontaneous breathing trial with automatic tube compensation versus continuous positive airway pressure. Crit Care Med, 2006;34:682-686.

25. Lellouche F, Mancebo J, Jolliet $P$ et al - A multicenter randomized trial of computer-driven protocolized weaning from mechanical ventilation. Am J Respir Crit Care Med, 2006;174:894-900.

26. Hendrix $\mathrm{H}$, Kaiser ME, Yusen $\mathrm{RD}$ et al - A randomized trial of automated versus conventional protocol-driven weaning from mechanical ventilation following coronary artery bypass surgery. Eur J Cardiothorac Surg, 2006;29:957-963.

27. Marini $\mathrm{JJ}$ - What derived variables should be monitored during mechanical ventilation? Respir Care, 1992;37:1097-1107.

28. Brown BR - Understanding mechanical ventilation: patient monitoring, complications, and weaning. J Okla State Med Assoc, 1994;87:411418.

29. Esteban A, Alia I - Clinical management of weaning from mechanical ventilation. Intensive Care Med, 1998;24:999-1008.

30. Jounieaux V, Duran A, Levi-Valensi P - Synchronized intermittent mandatory ventilation with and without pressure support ventilation in weaning patients with COPD from mechanical ventilation. Chest, 1994;105:12041210.

31. Esen F, Denkel T, Telci L et al - Comparison of pressure support ventilation (PSV) and intermittent mandatory ventilation (IMV) during weaning in patients with acute respiratory failure. Adv Exp Med Biol, 1992;317:371376.

32. HessD-Ventilatormodesusedinweaning.Chest,2001;120:(Suppl6):474S476S.

33. Epstein SK - What are the Best Methods for Weaning Patients from Mechanical Ventilation? Berlin Heidelberg: Springer-Verlag; 2004.

34. Meade M, Guyatt G, Cook D et al - Predicting success in weaning from mechanical ventilation. Chest, 2001;120:(Suppl6):400S-424S.

35. Meade MO, Guyatt GH, Cook DJ et al - Trials of corticosteroids to prevent postextubation airway complications. Chest, 2001;120:(Suppl6):464S468 S.

36. Ho LI, Harn HJ, Lien TC et al - Postextubation laryngeal edema in adults. Risk factor evaluation and prevention by hydrocortisone. Intensive Care Med, 1996;22:933-936.

37. Darmon JY, Rauss A, Dreyfuss D et al - Evaluation of risk factors for laryngeal edema after tracheal extubation in adults and its prevention by dexamethasone. A placebo-controlled, double-blind, multicenter study. Anesthesiology, 1992;77:245-251.

38. Chaney MA, Nikolov MP, Blakeman B et al - Pulmonary effects of methylprednisolone in patients undergoing coronary artery bypass grafting and early tracheal extubation. Anesth Analg, 1998;87:27-33.

39. Epstein SK, Ciubotaru RL - Independent effects of etiology of failure and time to reintubation on outcome for patients failing extubation. Am J
Respir Crit Care Med, 1998;158:489-493.

40. Miller RL, Cole RP - Association between reduced cuff leak volume and postextubation stridor. Chest, 1996;110:1035-1040.

41. Fisher MM, Raper RF - The 'cuff leak' test for extubation. Anaesthesia, 1992;47:10-12.

42. Engoren $\mathrm{M}$ - Evaluation of the cuff-leak test in a cardiac surgery population. Chest, 1999;116:1029-1031.

43. Khamiees M, Raju P, DeGirolamo A et al - Predictors of extubation outcome in patients who have successfully completed a spontaneous breathing trial. Chest, 2001;120:1262-1270.

44. Vallverdu I, Calaf N, Subirana M et al - Clinical characteristics, respiratory functional parameters, and outcome of a two-hour T-piece trial in patients weaning from mechanical ventilation. Am J Respir Crit Care Med, 1998;158:1855-1862.

45. Loudermilk EP, Hartmannsgruber M, Stoltzfus DP et al - A prospective study of the safety of tracheal extubation using a pediatric airway exchange catheter for patients with a known difficult airway. Chest, 1997; $111: 1660-1665$

46. Bauer TT, Ferrer R, Angrill J et al - Ventilator-associated pneumonia: risk factors, and microbiology. Semin Respir Infect, 2000;15:272-279.

47. Diehl JL, Atrous S, Touchard D et al - Changes in the work of breathing induced by tracheotomy in ventilator-dependent patients. Am J Respir Crit Care Med, 1999;159:383-338.

48. Lin MC, Huang CC, Yang CT et al - Pulmonary mechanics in patients with prolonged mechanical ventilation requiring tracheostomy. Anaesth Intensive Care, 1999;27:581-585.

49. Maziak DE, Meade MO, Todd TR - The timing of tracheotomy: a systematic review. Chest, 1998;114:605-609.

50. Rumbak MJ, Newton M, Truncale $T$ et al - A prospective, randomized, study comparing early percutaneous dilational tracheotomy to prolonged translaryngeal intubation (delayed tracheotomy) in critical ill medical patients. Crit Care Med, 2004;32:1689-1694.

51. Le Bourdelles G, Mier L, Fiquet B et al - Comparison of the effects of heat and moisture exchangers and heated humidifiers on ventilation and gas exchange during weaning trials from mechanical ventilation. Chest, 1996;110:1294-1298.

52. Girault C, Breton L, Richard JC et al - Mechanical effects of airway humidification devices in difficult to wean patients. Crit Care Med, 2003;31:1306-1311.

53. Knox JB, Wilmore DW, Demling $\mathrm{RH}$ et al - Use of growth hormone for postoperative respiratory failure. Am J Surg, 1996;171:576-580.

54. Felbinger TW, Suchner U, Goetz AE et al - Recombinant human growth hormone for reconditioning of respiratory muscle after lung volume reduction surgery. Crit Care Med, 1999;27:1634-1638.

55. Pichard C, Kyle U, Chevrolet JC et al - Lack of effects of recombinant growth hormone on muscle function in patients requiring prolonged mechanical ventilation: a prospective, randomized, controlled study. Crit Care Med, 1996;24:403-413.

56. Hebert PC, Blajchman MA, Cook DJ et al - Do blood transfusions improve outcomes related to mechanical ventilation? Chest, 2001;119:18501857.

57. al-Saady NM, Blackmore CM, Bennett ED - High fat, low carbohydrate, enteral feeding lowers $\mathrm{PaCO}_{2}$ and reduces the period of ventilation in artificially ventilated patients. Intensive Care Med, 1989;15:290-295.

58. van den Berg B, Bogaard JM, Hop WC - High fat, low carbohydrate, enteral feeding in patients weaning from the ventilator. Intensive Care Med, 1994;20:470-475. 